\title{
Peroral endoscopic myotomy for Jackhammer esophagus: to cut or not to cut the lower esophageal sphincter
}

Authors

Institutions
Robert Bechara ${ }^{1,2}$, Haruo lkeda ${ }^{2}$, Haruhiro Inoue ${ }^{2}$

Showa University - Digestive Diseases Center, Koto-Toyosu Hospital, Tokyo Japan

Queens University - Kingston General Hospital and Hotel Dieu Hospital Division of Gastroenterology, Kingston Ontario, Canada submitted 20. October 2015 accepted after revision 21. February 2016

\section{Bibliography}

Dol http://dx.doi.org/ 10.1055/s-0042-105204 Published online: 8.4.2016 Endoscopy International Open 2016; 04: E585-E588

(c) Georg Thieme Verlag KG Stuttgart . New York E-ISSN 2196-9736

\section{Corresponding author}

\section{Robert Bechara}

Showa University - Digestive

Diseases Center

Koto-Toyosu Hospital

5-1-38 Toyosu, Koto-ku

Tokyo Japan. koto

Please Select 135-8577

Japan

Fax: +81-3-6204-6064

bechara.robert@gmail.com
Background and study aims: With the success of peroral endoscopic myotomy (POEM) in treatment of achalasia, its successful application to other spastic esophageal motility disorders such as Jackhammer esophagus has been noted. The question of whether the lower esophageal sphincter (LES) should be included in the myotomy for Jackhammer esophagus is a topic of current debate. Here, we report our experience and results with four patients with Jackhammer esophagus treated with POEM. The clinical and manometric results are presented and their potential implications are discussed.

Patients and methods: Between January 2014 and July 2015, four patients underwent POEM for treatment of Jackhammer esophagus at our center. Manometry was performed prior to and after POEM. All patients met the Chicago classification criteria for Jackhammer esophagus and received a barium esophagram and endoscopic examination before having POEM.

\section{Introduction \\ $\nabla$}

Hypercontractile esophagus, previously referred to as "nutcracker esophagus," was defined manometrically by a mean distal contraction amplitude of $180 \mathrm{mmHg}$. This definition was plagued by a lack of specificity and poor symptom correction [1,2]. With the introduction of high-resolution manometry (HRM) in 2000, there have been changes to the manometric criteria for hypercontractile esophagus. The most recent definition is a distal contractile integer (DCI) of $\geq 8000 \mathrm{mmHg}$.cm.s in $\geq 20 \%$ of swallows and has been coined "Jackhammer esophagus." With this definition, there is improved specificity and symptom correlation compared with the previous criteria with standard manometry [3]. Jackhammer esophagus is rare, occurring in approximately $4 \%$ of cases referred to a tertiary esophageal center [4]. The treatment of Jackham-
Results: All patients had uneventful procedures without any intraoperative or post-procedure complications. Patients in which the LES was included during POEM had resolution or significant improvement in symptoms. One patient in whom the LES was preserved had resolution of chest pain but developed significant dysphagia and regurgitation. Subsequently this individual received a repeat POEM which included the LES, resulting in symptom resolution.

Conclusions: POEM is a suitable treatment for patients with Jackhammer esophagus. Until there are larger-scale randomized studies, we speculate that based on our clinical experience and physiologic and manometric observations, obligatory inclusion of the LES is justified to reduce the risk of symptom development from iatrogenic ineffective esophageal motility or subsequent progression to achalasia.

mer esophagus has included oral nitrates, balloon dilation, and surgical myotomy [5]. Surgical myotomy has not been widely performed due to the usual requirement for a long myotomy to achieve clinical success, which generally necessitates a combined abdominal and thoracic approach if a complete myotomy of the LES is to be performed [6-8]. With peroral endoscopic myotomy (POEM), a long myotomy is possible without increased morbidity or technical difficulty. However, experience with POEM for Jackhammer esophagus has been limited due to the rarity of the disorder. In addition, the inclusion/exclusion of the lower esophageal sphincter (LES) in the myotomy is debated and variably performed, perhaps contributing to inconsistent clinical outcomes in Jackhammer esophagus [911]. POEM was performed on four patients with Jackhammer esophagus at our center. Here we 
Table 1 Patients with Jackhammer esophagus treated with POEM

\begin{tabular}{|c|c|c|c|c|c|c|c|c|c|}
\hline \multirow[t]{2}{*}{ Patient } & \multirow[t]{2}{*}{ Myotomy (cm) } & \multirow[t]{2}{*}{ LES included } & \multicolumn{2}{|c|}{ Median IRP (mmHg) } & \multicolumn{2}{|c|}{ Mean DCl (mmHg.cm.s) } & \multicolumn{2}{|c|}{ Eckardt score } & \multirow[t]{2}{*}{ IEM* } \\
\hline & & & Before & After & Before & After & Before & After & \\
\hline 1 & 20 & - & 19.5 & 23.5 & 12516.5 & 84.2 & 2 & 6 & + \\
\hline 2 & 21 & + & 16.4 & 10.5 & 18332.4 & 137.7 & 5 & 0 & + \\
\hline 3 & 12 & + & 33.8 & 16.2 & 46700 & 2019.6 & 5 & 0 & - \\
\hline 4 & 23 & + & 7.3 & 12.4 & 15388.7 & 234 & 11 & 2 & + \\
\hline
\end{tabular}

* IEM-ineffective esophageal motility after POEM $=\geq 50 \%$ ineffective swallows (failed or weak contraction vigor $[\mathrm{DCl}<450 \mathrm{mmHg} . \mathrm{cm} . \mathrm{s}]$ )

will present the clinical and manometric results and discuss the treatment implications.

\section{Patients and methods \\ $\nabla$}

Between January 2014 and July 2015, four patients underwent POEM for treatment of Jackhammer esophagus at our center. Written informed consent for POEM was obtained from all patients. In this series, data was prospectively collected and retrospectively examined. All patients had undergone a trial of at least 2 weeks on a proton pump inhibitor (PPI) without improvement in symptoms. Manometry was performed using the Starlet ${ }^{\circledR}$ system (Star Medical, Tokyo, Japan) (Normal integrated relaxation pressure [IRP] for Starlet ${ }^{\circledR}$ system $25 \mathrm{mmHg}$ [12]) prior to and 2 months after POEM. The diagnosis of Jackhammer esophagus was based on the manometric criteria stated above. All patients met the criteria for Jackhammer esophagus and received a barium esophagram and endoscopic examination prior to POEM. Furthermore, one patient (Patient 3 ) received a computed tomography (CT) scan because of elevated IRP in addition to a distal contractile integral (DCI) $\geq 8000 \mathrm{mmHg} . \mathrm{cm} . \mathrm{s}$ in $\geq 20 \%$ of swallows. Follow-up was performed every 3 months for the first year then annually thereafter or sooner if issues developed.

The POEM procedure was performed as previously described [13]. Briefly, a submucosal bleb was created with saline and indigo carmine. The point at which the tunnel was started was based on the manometry, barium esophagram, and endoscopic examination. The objective was to start the myotomy at the most proximal extent of the hypertensive contractions. A 2- to 3-cm longitudinal mucosal incision was made, submucosal entry achieved, and the submucosal tunnel was created. When the LES was included in the myotomy, the tunnel was advanced 2 to $3 \mathrm{~cm}$ into the gastric cardia. The myotomy was advanced from 1 to $2 \mathrm{~cm}$ distal to the mucosal incision to the distal end of the tunnel. However, when the LES was not included the tunnel the myotomy was only advanced to the distal esophagus. After prophylactic antibiotics were instilled, the mucosal entry site was closed with hemostatic clips. On Day 1 post-procedure, all patients received a barium esophagram and endoscopy to confirm the mucosal integrity. The diet was advanced over 4 days and patients were discharged on day 4 post-procedure.

\section{Results \\ $\nabla$}

The manometric and clinical results are summarized in $\bullet$ Table 1 and details about each patient are described below. All patients had uneventful procedures without any intraoperative or postprocedure complications. Esophageal manometry, upper endoscopy, and clinical (Eckardt score, gastroesophageal reflux symp- toms) examinations were performed 2 months post-POEM. No patients developed clinical or endoscopic evidence of reflux.

\section{Patient 1}

A 74-year-old man presented with a 15-year history of weekly to daily severe noncardiac chest pain associated with swallowing. An endoscopy revealed no mucosal pathology and a small epiphrenic diverticulum. Manometry exhibited multi-peaked contractions with a mean DCI of $12516.5 \mathrm{mmHg} . \mathrm{cm} . \mathrm{s}$ and median IRP of $19.5 \mathrm{mmHg}(\boldsymbol{B}$ Fig. 1). A 20-cm myotomy was performed, sparing the LES due to lack of its involvement in the abnormal contractions. The patient's chest pain completely resolved, but he developed frequent dysphagia and regurgitation (Eckardt score 6). Six months later, the patient underwent a second POEM with a 10-cm myotomy that included the LES, resulting in the resolution of dysphagia and regurgitation (the patient chose to forego repeat manometry after the second POEM). Clinical follow-up was achieved for a total of 18 and 6 months after the first and second POEM's, respectively. ( $\mathbf{O}$ Fig. $\mathbf{1}$ )

\section{Patient 2}

A 68-year-old man with a 33-year history of regurgitation and dysphagia presented with progression of symptoms and weight loss (Eckardt score 5). Manometry demonstrated a mean DCI of $18332.4 \mathrm{mmHg} . \mathrm{cm} . \mathrm{s}$ and a median IRP of $16.4 \mathrm{mmHg}$ with inclusion of the LES in the hypercontractile segment ( $\boldsymbol{F}$ Fig.2). The patient received a POEM with a 21-cm myotomy that included the LES.After POEM, the patient's symptoms completely resolved (Eckardt score 0). The follow-up HRM demonstrated a DCI of $137.7 \mathrm{mmHg} . \mathrm{cm} . \mathrm{s}$ and a median IRP of $10.5 \mathrm{mmHg}$. Clinical follow-up was achieved for a total of 12 months after POEM.( $\bullet$ Fig. 2 )

\section{Patient 3}

An 87-year-old man with a 40-year history of dysphagia presented with symptom progression leading to weight loss (Eckardt score 5). Manometry demonstrated a mean DCI of $46700 \mathrm{mmHg}$. $\mathrm{cm} . \mathrm{s}$ with a median IRP $33.8 \mathrm{mmHg}$ with the LES included in the hypercontractile segment ( $\bullet$ Fig.3). A CT scan was also performed in light of the elevated IRP, which did not reveal any infiltrative, neoplastic or vascular obstruction at the distal esophagus. The patient received a $12-\mathrm{cm}$ myotomy that included the LES, which resulted in complete symptom resolution (Eckardt score 0 ). Clinical follow-up was achieved for a total of 12 months after POEM ( $\bullet$ Fig. 3 )

\section{Patient 4}

A 37-year-old man with a 6-year history of dysphagia, regurgitation, chest pain presented with deterioration of symptoms resulting in weight loss (Eckardt score 11). HRM showed DCI of $15388.7 \mathrm{mmHg} . \mathrm{cm} . \mathrm{s}$ and median IRP $7.3 \mathrm{mmHg}(\boldsymbol{\bullet}$ Fig. 4 ). The patient received a POEM with a $23-\mathrm{cm}$ myotomy that included the 

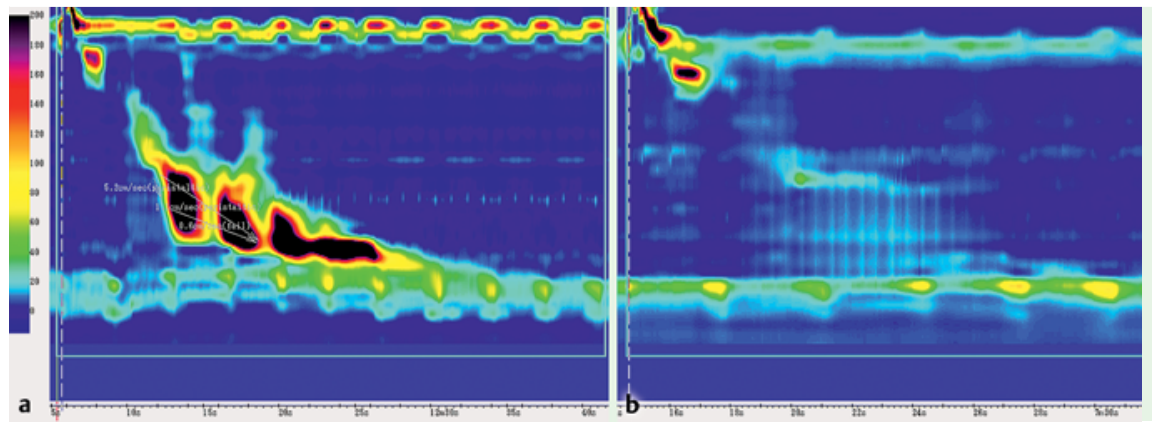

Fig. 1 Patient 1 HRM pre- and post-POEM \#1. a Multi-peaked contractions with $\mathrm{DCl}$ of $12516.5 \mathrm{mmHg} . \mathrm{cm} . \mathrm{s}$ and median IRP of $19.5 \mathrm{mmHg}$. b Post first POEM showing a lack of abnormal contractions with failed contraction vigor with a mean $\mathrm{DCl}$ of $84.2 \mathrm{mmHg} . \mathrm{cm} . \mathrm{s}$ and median IRP of $23.2 \mathrm{mmHg}$.

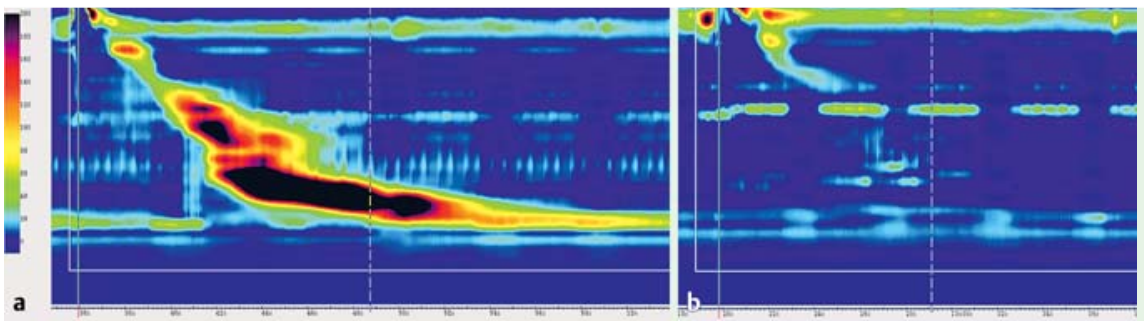

Fig. 2 Patient 2 HRM pre and post-POEM. a Hypercontractile contractions with a mean DCI of $18332.4 \mathrm{mmHg} . \mathrm{cm} . \mathrm{s}$ and median IRP of $16.4 \mathrm{mmHg}$ b Post-POEM with no abnormal contractions with a weak contraction vigor with a mean $\mathrm{DCl}$ of $137.7 \mathrm{mmHg} . \mathrm{cm} . \mathrm{s}$ and median IRP of $10.5 \mathrm{mmHg}$.

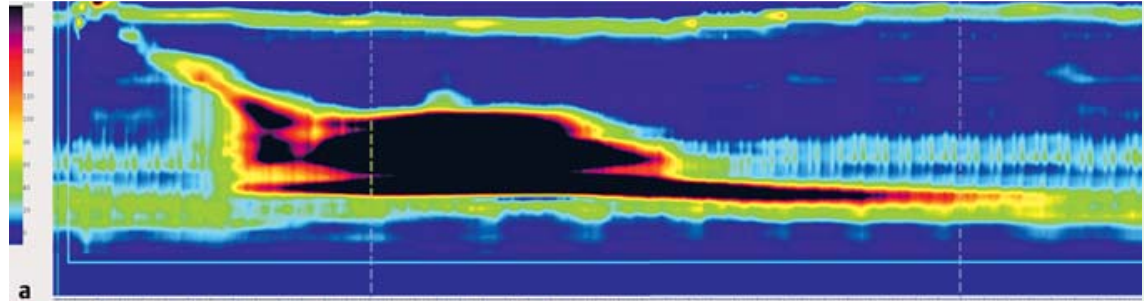

Fig. 3 Patient 3 HRM pre- and post-POEM a Hypercontractile contractions with a mean $\mathrm{DCl}$ of $46700 \mathrm{mmHg} . \mathrm{cm} . \mathrm{s}$ and median IRP $33.8 \mathrm{mmHg}$ b Post-POEM showing no abnormal contractions and a normal contraction vigor with mean $\mathrm{DCl}$ of $2019.6 \mathrm{mmHg} . \mathrm{cm} . \mathrm{s}$ and median IRP $16.2 \mathrm{mmHg}$.
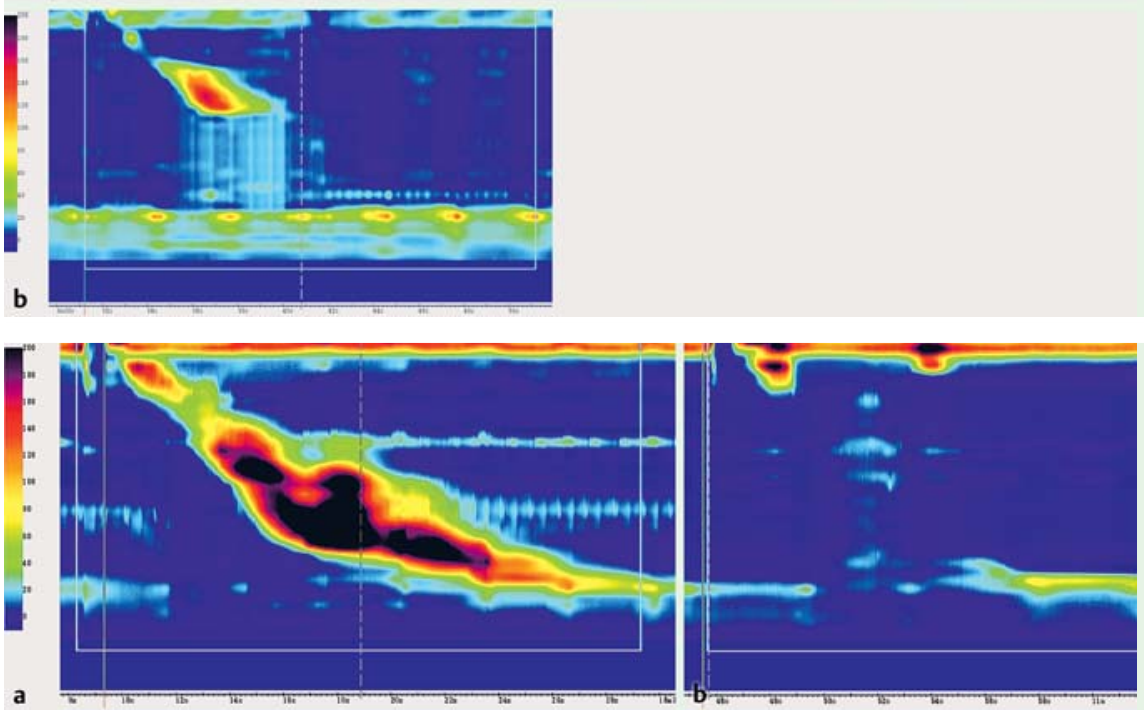

Fig. 4 Patient 4 HRM pre- and post-POEM a Hypercontractile contractions with a mean $\mathrm{DCl}$ of $15388.7 \mathrm{mmHg} . \mathrm{cm} . \mathrm{s}$ and median IRP $7.3 \mathrm{mmHg}$ b Post-POEM showing no abnormal contractions with a weak contraction vigor with a mean $\mathrm{DCl}$ of $234 \mathrm{mmHg} . \mathrm{cm} . \mathrm{s}$ and median IRP $12.4 \mathrm{mmHg}$.

LES. After POEM, the patient regained the weight he had lost and his chest pain completely resolved with only occasional dysphagia and regurgitation (Eckardt score 2). Clinical follow-up was achieved for a total of 12 months after $\operatorname{POEM}(\bullet$ Fig.4) $(\bullet$ Table 1$)$.

\section{Discussion}

$\nabla$

With inclusion of the LES in POEM for Jackhammer esophagus, Patients 2, 3, and 4 had excellent clinical results. In contrast, Patient 1 in whom LES was not included in the myotomy developed regurgitation and dysphagia. However, after the second POEM that included the LES, his symptoms of dysphagia and regurgitation resolved. With Patient 1, the HRM was consistent with
Jackhammer esophagus, with a mean DCI of $12516.5 \mathrm{mmHg} . \mathrm{cm} . \mathrm{s}$ and a normal IRP. However, the IRP was in the upper range of normal and one could speculate that the patient was progressing to achalasia and had a variant of "incompletely expressed" or "early" achalasia.

The progression from hypercontractile esophagus or diffuse esophageal spasm (DES) to achalasia has previously been described, suggesting that the spastic esophageal motor disorders may represent a spectrum of a single disease entity $[14,15]$. Interestingly, there have been no reports of progression from one spastic disorder to another demonstrated with HRM. This may be due to the previous misdiagnosis with standard manometry, the superior sensitivity and specificity of HRM, and the new Chicago classification. That, on the other hand, would support the 
notion that the spastic esophageal motor disorders are separate entities rather than a spectrum of a single pathology. The successful treatment of Jackhammer esophagus usually requires a long myotomy, often two-thirds or more the length of the esophagus, which can often result in iatrogenic ineffective esophageal motility [16]. This was demonstrated in our series by the fact that all patients with a myotomy $20 \mathrm{~cm}$ or longer developed ineffective esophageal motility ( $\geq 50 \%$ ineffective swallows), while the patient with a 12-cm myotomy had persevered contraction vigor. Without involvement of the LES in the hypercontractile segment, inclusion of LES in the myotomy has been a contentious topic. However, we postulate that routine inclusion of the LES in POEM for Jackhammer esophagus should be performed for the following reasons:

1. Patients with Jackhammer esophagus typically require a long myotomy, which results in diminished contraction vigor and in many cases iatrogenic ineffective esophageal motility. In some patients, gravity and the remaining propulsive force is inadequate to propagate the food bolus across the preserved LES. Without the inclusion of the LES in the myotomy to further reduce outflow resistance, symptoms analogous to achalasia may develop (regurgitation, dysphagia, and chest pain). Thus, there is a critical (currently unknown) length of esophageal myotomy that once exceeded, results in ineffective esophageal motility, inadequate food bolus propulsion, and symptom development. The importance of LES inclusion was demonstrated in a case report by Badillo et al., in which a 50-year-old woman received POEM for Jackhammer esophagus, resulting in worsening symptoms post-POEM. She subsequently presented with continued deterioration in symptoms and was found to have an $8-\mathrm{cm}$ anterolateral diverticulum with moderate narrowing of the gastroesophageal junction. [17] Based on her immediate post-POEM symptom deterioration and subsequent development of the diverticulum, it is highly likely the LES was not included in the myotomy analogous to, although more dramatic, than our Patient 1.

2. There is evidence that the non-achalasia spastic esophageal motility disorders can progress to achalasia. Albeit rare, if there is progression to achalasia and the LES is preserved, symptom development would occur and the patient would require additional treatment.

3. The risk of complicated reflux developing due to myotomy of the LES is negligible. Although there is indeed a significant risk of reflux after POEM (up to 50\%), there are no cases of reflux refractory to PPI, and only one report of a peptic stricture. [18-20]

In conclusion, POEM is a suitable treatment for patients with Jackhammer esophagus. Based on our clinical experience and physiologic and manometric observations, we speculate that the obligatory inclusion of the LES is justified. Inclusion of the LES minimizes the risk of symptom development from iatrogenic ineffective esophageal motility or subsequent progression to achalasia. Furthermore, from our experience, in addition to the thousands of cases of POEM published, the risk of reflux-related complications has been shown to be marginal. Thus, it appears that the risks associated with LES exclusion are far greater than the risks of reflux-associated complications of LES inclusion. However, given the low incidence of Jackhammer esophagus, an international, multicenter randomized trial is required in order to obtain a definitive evidence-based answer to whether routine inclusion/exclusion of the LES in the application of POEM for Jackhammer esophagus is warranted.

\section{Competing interests: None}

\section{References}

1 Jung KW, Jung HY, Yoon IJ et al. New diagnostic criteria for nutcracker esophagus using conventional water-perfused manometry: a comparison between nutcracker esophagus with and without gastroesophageal reflux disease. Journal of gastroenterology and hepatology 2010; 25: $1239-1243$

2 Roman $S$, Tutuian $R$. Esophageal hypertensive peristaltic disorders. Neurogastroenterology and motility: the official journal of the European Gastrointestinal Motility Society 2012; 24: 32 - 39

3 Kahrilas PJ, Bredenoord AJ, Fox $M$ et al. The Chicago Classification of esophageal motility disorders, v3.0. Neurogastroenterology and motility: the official journal of the European Gastrointestinal Motility Society 2015; 27: $160-174$

4 Roman S, Pandolfino JE, Chen J et al. Phenotypes and clinical context of hypercontractility in high-resolution esophageal pressure topography (EPT). Am J Gastroenterol 2012; 107: 37-45

5 Valdovinos MA, Zavala-Solares MR, Coss-Adame E. Esophageal hypomotility and spastic motor disorders: current diagnosis and treatment. Curr Gastroenterol Rep 2014; 16: 421

6 Patti MG, Gorodner MV, Galvani C et al. Spectrum of esophageal motility disorders: Implications for diagnosis and treatment. Archives of Surgery 2005; 140: 442 - 449

7 Ellis FH Jr. Esophagomyotomy for noncardiac chest pain resulting from diffuse esophageal spasm and related disorders. The American journal of medicine 1992; 92: 129s-131s

8 Coss-Adame E, Erdogan A, Rao SS. Treatment of esophageal (noncardiac) chest pain: an expert review. Clinical gastroenterology and hepatology : the official clinical practice journal of the American Gastroenterological Association 2014; 12: $1224-1245$

9 Khashab MA, Saxena P, Kumbhari Vet al. Peroral endoscopic myotomy as a platform for the treatment of spastic esophageal disorders refractory to medical therapy (with video). Gastrointestinal endoscopy 2014; 79: 136 - 139

10 Almansa $C$, Hinder RA, Smith CD et al. A comprehensive appraisal of the surgical treatment of diffuse esophageal spasm. Journal of gastrointestinal surgery: official journal of the Society for Surgery of the Alimentary Tract 2008; 12: $1133-1145$

11 Herbella FA, Tineli AC, Wilson JL Jr et al. Surgical treatment of primary esophageal motility disorders. Journal of gastrointestinal surgery: official journal of the Society for Surgery of the Alimentary Tract 2008; 12 : 604-608

12 Kuribayashi S, Iwakiri K, Kawada A et al. Variant parameter values-as defined by the Chicago Criteria-produced by ManoScan and a new system with Unisensor catheter. Neurogastroenterology and motility: the official journal of the European Gastrointestinal Motility Society 2015; 27: $188-194$

13 Inoue $H$, Minami $H$, Kobayashi $Y$ et al. Peroral endoscopic myotomy (POEM) for esophageal achalasia. Endoscopy 2010; 42: 265-271

14 Paterson WG, Beck IT, Da Costa LR. Transition from nutcracker esophagus to achalasia. A case report. Journal of clinical gastroenterology 1991; $13: 554-558$

15 Fontes LH, Herbella FA, Rodriguez TN et al. Progression of diffuse esophageal spasm to achalasia: incidence and predictive factors. Diseases of the esophagus: official journal of the International Society for Diseases of the Esophagus / ISDE 2013; 26: 470-474

16 Patti MG, Pellegrini CA, Arcerito $M$ et al. Comparison of medical and minimally invasive surgical therapy for primary esophageal motility disorders. Archives of surgery (Chicago, Ill: 1960) 1995; 130: 609615; discussion 615-606

17 Badillo R, Francis D, DeVault K. Formation of large esophageal diverticulum after peroral endoscopic myotomy. Gastrointestinal endoscopy 2015: DOI 10.1016/j.gie.2015.05.020

18 Werner YB, Costamagna G, Swanstrom LL et al. Clinical response to peroral endoscopic myotomy in patients with idiopathic achalasia at a minimum follow-up of 2 years. Gut 2015: DOI 10.1136/gutjnl-2014308649

19 Bechara R, Ikeda H, Inoue H. Peroral endoscopic myotomy: an evolving treatment for achalasia. Nat Rev Gastroenterol Hepatol 2015; 12: 410-426

20 Familiari P, Greco S, Gigante G et al. Gastroesophageal reflux disease after peroral endoscopic myotomy: Analysis of clinical, procedural and functional factors, associated with gastroesophageal reflux disease and esophagitis. Digestive Endoscopy 2016; 28: 33 -41 